\title{
OF THE RELATIONSHIP OF LAW AND TECHNOLOGY
}

Rubén Toledo ORIHUELA*

Andrés Saavedra AVENDAÑO**

\begin{abstract}
This work seeks to present the difference between computer law and legal information; that is, a vision of law from the perspective of a technology at the service of societies. At present it is fused with computer science tools that places us in the possibility of improving the effectiveness of the work of law, which is the search for values such as justice, legal security and the common good. The above opens a new knowledge gap that can explain a relationship between the law and the aforementioned values in a more transparent, quick and accurate way.
\end{abstract}

Keywords: Law; Computer Law; Legal Informatics and use of technologies

DOI: 53373 / REDS.2021.53.4.048

Los temas del presente ensayo se trataran en el siguiente orden: 1. Distinción entre derecho informático e informática jurídica 2. Posturas del uso de tecnologías en el derecho, y 3. La ética y lógica en el debate del uso de las tecnologías en el derecho.

\section{DISTINCIÓN ENTRE DERECHO INFORMÁTICO E INFORMÁTICA JURÍDICA}

La perspectiva de estas reflexiones son sin duda la herramienta del pensamiento con la que podremos interpretar un trabajo, en este caso es importante recalcar que no nos adentraremos al estudio del derecho en su sentido tradicionalmente conocido, ni mucho menos veremos la filología tan debatida en el mundo humano desde su concepción como tecnología de control social, por ello se concibe este tema desde el punto de vista del uso del derecho mismo.

"El derecho mismo - sus categorizaciones, sus procedimientos, sus prácticasconstituye una tecnología cognitiva, un modo de aproximarse a la realidad social para controlarla, dirigirla y hacer efectivos en ella determinados planes y modelos económicos, sociales y políticos." 1

* Profesor Investigador de la Facultad de Derecho y Ciencias Sociales de la Universidad Autónoma del estado de Morelos, México.

** Estudiante del Doctorado en Derecho y Globalización. PNPC-Conacyt. Facultad de Derecho y Ciencias Sociales de la Universidad Autónoma del estado de Morelos, México.

1 Casanovas. Pompeu, Derecho, Tecnología, Inteligencia Artificial Y Web Semántica. Un Mundo Para Todos Y Para Cada Uno" Texto extraído del Portal Web de la Universidad Nacional Autónoma de México, Instituto de Investigaciones Jurídicas. https://biblio.juridicas.unam.mx/bjv/detalle-libro/3875enciclopedia-de-filosofia-y-teoria-del-derecho-volumen-uno. 
El derecho no puede verse más desde una sola perspectiva, solo como conjunto de normas o planteamientos hipotéticos debidamente legislados, ni mucho menos como el intangible uso de la justicia ante determinada población y espacio territorial; considero que la era de los procedimientos sociales revolucionarios ha comenzado y la tecnología es una herramienta que hoy comienza a ser la antorcha incendiaria en la propagación de los nuevos movimientos sociales, prueba reciente son las grandes protestas en Latinoamérica y en Europa, los movimientos feministas con demandas de igualdad de género, con coreografías y reclamos casi exactamente iguales en un continente que en otro.

La concepción tradicional vinculada con el imperium ${ }^{2}$ no contraviene la perspectiva del presente trabajo, ya que si bien encontramos unión entre el derecho, desde su concepción de conjunto normativo, más vinculado con sus fines epistemológicos, lo cierto es que, ver los mecanismos tecnológicos y sociales que hoy día rodean a la humanidad, para precisamente hacer cumplir este fin, no escapa de su significado mismo, es tan solo la distinción del fin que tiene el derecho y cuál es precisamente, el procedimiento para hacerlo cumplir con mayor efectividad.

El derecho ha evolucionado en su aspecto no solo normativo, sino también en cuanto a la forma de desarrollarse en la vida social, y la sociedad actual como las pasadas fueron y seguirán evolucionando, el cambio, llamado devenir constante de la sociedad, ${ }^{3}$ desde las reflexiones y el enfoque ético de la filosofía de Friedrich Nietzsche; es parte de esta historia sin fin, en donde el derecho jamás se ha quedado atrás, desde la regulación en piedras, en lugares sagrados, en pergaminos, en papel y tinta, en impresiones y hoy, consultable en nuestros smartphones, el futuro ya está aquí y la herramienta es la informática, las computadoras, los sistemas son hoy el papel que escribe la historia de cómo se comportará el derecho para lograr sus fines.

Ahora bien y con la intención de establecer parámetros respecto de nuestras reflexiones, debemos señalar que ésta no pretende adentrarse en lo profundo de la regulación de la tecnología, ni en reglamentar o emitir leyes entorno al uso de la informática, o los millones de datos que diariamente circulan a gran velocidad por la $\boldsymbol{w e b}$, por lo que cabe precisar que este artículo, se concentrará en poder establecer el vínculo lingüístico que rige al derecho hoy día.

Para ello debemos primeramente establecer una distinción entre el derecho informático e la informática jurídica, esto para posicionar nuestro tema en una rama del derecho en particular, y con ello aclarar la perspectiva teórica que se pretende al

\footnotetext{
${ }^{2}$ Este término latino guarda relación con la potestad del Estado de hacer cumplir sus determinaciones, en la antigua Roma se otorgaba esta potestad disciplinaria a los pretores, para muchos es precisamente el puño del Estado que con fuerza puede hacer cumplir sus determinaciones con el elemento clave de distinción del derecho de otros "listados normativos" como el moral o el religioso, el carácter de obligatoriedad y el resultado de su omisión o acción en contra de las normativas de cada Estado.

3 "La forja de una identidad ética en el pensamiento de Nietzsche", Alba Elizabeth Huitrón Villegas alba.huitron@itesm.mx Instituto Tecnológico y de Estudios Superiores de Monterrey, México; En-claves del pensamiento, vol. XII, núm. 23, 00001, 2018; Instituto Tecnológico y de Estudios Superiores de Monterrey, División de Humanidades y Ciencias Sociales
} 
abordarla, y desde una filosofía del derecho "contiene reflexiones acerca del concepto de derecho, la controvertida estructura del fenómeno jurídico, el modo de relacionarlo con la realidad social y, según muchos, la manera de vincular los temas anteriores con el arte de distinguir lo bueno de lo malo y lo justo de lo injusto". ${ }^{4}$

$\mathrm{Y}$ es que desde la superficie pareciera que no existe una distinción entre los conceptos del derecho en su análisis teórico y en la forma en que se elabora la tecnología social que lo hace efectivo, el vínculo que tiene con una colectividad, en este sentido las computadoras en la actualidad "realizan el manejo de información" como se había hecho ya con anterioridad en los números de expedientes, los afiches, la doctrina escrita y consultable en lugares específicos, inclusive la compilación jurídica llamada de..."Las Doce Tablas podría identificarse como un gran hito en la informática jurídica occidental..."

La tecnología para Hendrik Van Loon: ”...puede verse como prolongaciones del Cuerpo Humano. Así, el pie se extiende al carro, a la bicicleta, al ferrocarril, al automóvil, al avión... el ojo en el microscopio y el telescopio... el cerebro se ve prolongado en las actuales máquinas de pensar...".

Para comprender un poco más de lo que se habla, es que la informática jurídica se propone como "...el saber, cómo el hacer algo y no solo saber hacerlo, saber hacer algo (ser capaz de hacerlo en la práctica), y saber cómo hacer algo (ser capaz de explicar los pasos y criterios que, debidamente empleados, conduzcan con seguridad al fin deseado). ${ }^{6}$

La informática jurídica tiene que ver con el dominio técnico de una forma de hacer determinados actos, en el caso de los sistemas obedecen a las instrucciones dadas por el programador, en donde se pretende poder responder con la mayor exactitud en el menor tiempo y de forma más eficiente a cualquier tipo de determinación que se le proponga al sistema predeterminadamente.

El derecho es una concepción en el mundo social y no como ciencia del espíritu, claro que tecnológicamente se respeta la perspectiva epistémica de su concepto en el mundo de lo humano, lo cierto es que los sistemas son precisamente programaciones que tienen enfoques humanos, y que requieren de un conocimiento para su implantación en las sociedades y su uso cotidiano, este fenómeno puede ser meramente técnico, es decir, solo por la forma de obtener el resultado esperado, esto puede verse desde dos perspectivas una abierta y otra cerrada.

Julia Barragán parece expresar conformidad con la orientación epistemológica tradicional: el jurista tiene derecho a su propia forma de razonar, que incluye la textura abierta del lenguaje y desemboca en más de una respuesta correcta para cada problema. ${ }^{7}$

\footnotetext{
${ }^{4}$ A. Guibourg, Ricardo. Informática Jurídica, UNAM, consultable en el portal web consultado con fecha: 4 Diciembre 2019 https://archivos.juridicas.unam.mx/www/bjv/libros/8/3875/25.pdf 
Daniele Bourciere, desde una óptica más especifica, puso de resalto que el uso de la informática jurídica es, como la codificación, un modo de racionalización de la producción jurídica". ${ }^{8}$

La informática jurídica por ende, se entendería como el uso de esta materia en toda su expresión para lograr aportar en la resolución o mejora en tiempo, exactitud y esfuerzo la actividad que rodea el cumplimiento del derecho como esencia social, hoy tiene un vínculo bastante fuerte con el quehacer humano, como el uso de la tecnología informática para la búsqueda de datos, mejora de la comprensión de determinados temas, soluciones más eficientes y las mil y un actividades que ahora aportan los sistemas a las actividades humanas.

Por otro lado, el derecho informático, tiene más que ver con la perspectiva del derecho como orden regulador de las conductas humanas, es decir que observa a la tecnología no como una vía sino, como un fenómeno a regular, es decir, que determina y delimita el uso de la tecnología para fines de regular su acción, y su presentación ante el Estado y la sociedad, para legitimar de su uso y sancionar su mal manejo.

"Vale decir que se entenderá el derecho informático como el tratamiento sistemático y normativo tendiente a regular la informática en sus múltiples aplicaciones (burocrática, robótica, telemática etc.).",

Otro de los temas importantes a definir es precisamente, la conjunción de los vocablos usados y nos referimos a la informática "el tratamiento automático de la información a través de elaboradores electrónicos basados en las reglas de la cibernética" 10 .

La informática "es el conjunto de conocimientos científicos y técnicos que hacen posible la resolución de forma automática de problemas por medio de las computadoras... La informática es, en definitiva, una rama del saber que abarca todos los aspectos del diseño y uso de las computadoras y como consecuencia, debería cubrir todos aquellos problemas con los que nos encontramos a la hora de realizar una tarea con una máquina de forma automática". ${ }^{11}$

La comprensión de la distinción entre el fin del derecho regulador y el proceso de dicha finalidad, unidas a la materia de resolución de problemas a través de las máquinas y de los sistemas informáticos, nos lleva a comprender que los Estados no solo regulan las actividades de las máquinas, sino también, se sirven de ellas para el

${ }^{8}$ Ibidem p. 813.

9 Rodríguez, Felipe. "Lecciones de derecho y ética para ingenieros, estudiantes de ingeniería y profesiones afines" UNC, consultable en su totalidad en el portal web www.feliperodriguez.com.ar/wpcontent/uploads/2013/11/LIBRO-7-DERECHO-INFORMATICO.pdf , consultado con fecha 4 de Diciembre de 2019.

${ }^{10}$ Cita tomada del texto antes señalado correspondiente al autor Gustavino, Elías "Responsabilidad civil y otros problemas jurídicos en computación”, Ed. La Rocca, 1987.

11 Fundamentos de Informática I, Conceptos generales, consultable en el portal web. https://www.infor.uva.es/ cevp/FI_I/fichs_pdf_teo/FI_I_Tema1.pdf, consultado con fecha 10 de Diciembre de 2019. 
mejoramiento de sus actividades propias, y la impartición de justicia de ninguna manera se escapa del uso de la tecnología para lograr sus fines.

\section{POSTURAS DEL USO DE TECNOLOGÍAS EN EL DERECHO}

"Lejos de ser ciencia-ficción, la inteligencia artificial (IA) forma ya parte de nuestras vidas. En la utilización de un asistente personal para organizar nuestra jornada laboral, en el desplazamiento en un vehículo de conducción automática o en las canciones o restaurantes sugeridos por nuestros teléfonos, la IA se hace realidad. ${ }^{, 12}$

En este sentido hoy día la inteligencia artificial se torna como un tema a debate de interés global, que lejos de ser una perspectiva de ciencia ficción, se convierte en una realidad que se vive a diario, y que poco a poco va permeando del uso militar, hacia el uso personal, y su direccionamiento hacia la implementación del derecho con fines de agilizar los trámites que por su cuenta corresponden al Estado, la forma en que los abogados llevan sus asuntos, los procedimientos de bastantes servicios en línea entre ellos incluso, los de asesoría legal.

El debate precisamente tiene un sesgo entorno a las aporías éticas y de responsabilidad del uso de las tecnologías para el mejoramiento de la calidad de vida humana, esto con la intención de un desarrollo adecuado de la inteligencia artificial, que se encuentre acorde con los valores y derechos fundamentales de sus usuarios, así como con la obligación de rendición de cuentas y transparencia que requiere cualquier política, sin dejar de lado, la concepción de un planeta como nuestro ambiente, en donde en el debate de la ponderación de los derechos, podríamos determinar que el derecho de nuestro hábitat, de la concepción del planeta como un ente que debe determinar nuestras actividades por nuestro fin mismo de supervivencia.

En razón de esto, los sistemas políticos más avanzados en la actualidad hacen uso ya de la digitalización, sistematización algorítmica y en algunos casos de inteligencia artificial para eficientar sus actividades, al caso vale la pena transcribir lo que estableció el Consejo Europeo ${ }^{13}$ en mayo de 2017 "la UE necesita concienciarse de la urgencia de hacer frente a las nuevas tendencias, tales como la IA, "garantizando al mismo tiempo un elevado nivel de protección de los datos, así

\footnotetext{
${ }^{12}$ Este fragmento forma parte del contenido de la Comisión Europea, en relación con la Inteligencia Artificial, dictado con fecha 25 de Abril de 2018, el cual puede ser consultado en el siguiente portal web, https://eur-lex.europa.eu/legal-content/ES/TXT/?uri=CELEX:52018DC0237, consultado con fecha 10 de Noviembre de 2019.

${ }^{13}$ Este ente tiene como finalidad Definir la orientación y las prioridades políticas generales de la Unión Europa, para mejor comprensión de dicho consejo se recomienda la búsqueda y seguimiento de dicho Consejo mediante su portal web oficial consultado con fecha 10 de noviembre de 2019. https://europa.eu/european-union/about-eu/institutions-bodies/european-council_es
} 
como los derechos digitales y las normas éticas», invitando a que «la Comisión [...] proponga un planteamiento europeo respecto de la inteligencia artificial»" "14

Es claro que el uso de las tecnologías de punta, han tenido y seguirán teniendo injerencia en las actividades diarias de la humanidad, situación que resulta perceptible, ya que si bien, su uso guarda relación actualmente con la potenciación de la capacidad tecnológica e industrial de la Unión Europea (UE), debemos recalcar que la tendencia en el estudio de las tecnologías, según la comisión de Inteligencia Artificial de la Unión, se ciñó en los siguientes puntos:

Potenciar la capacidad tecnológica e industrial de la UE e impulsar la adopción de la IA en todos los ámbitos de la economía, tanto en el sector privado como en el público.

Las inversiones en investigación e innovación y en la mejora del acceso a los datos forman parte de este objetivo.

Prepararse para las transformaciones socioeconómicas que origina la IA, fomentando la modernización de los sistemas de educación y formación, favoreciendo el talento, previendo los cambios en el mercado laboral y prestando apoyo a las transiciones que se operen en él y a la adaptación de los sistemas de protección social.

Garantizar el establecimiento de un marco ético y jurídico apropiado, basado en los valores de la Unión y en consonancia con la Carta de los Derechos Fundamentales de la UE. Incluye una próxima directriz sobre la interpretación de las actuales normas en materia de responsabilidad por productos defectuosos y un análisis pormenorizado de los retos emergentes, asi como la cooperación con las partes interesadas, en el seno de una Alianza europea de la IA, para elaborar directrices éticas en la materia. ${ }^{15}$

Con lo anterior podemos entender el interés actual entorno al uso de las tecnologías, y su complicación tiene que ver con cuestiones éticas y marcos jurídicos, por lo que derivado de que el trabajo tiene una relación netamente jurídica con la tecnología, corresponde proponer su análisis respecto de esta perspectiva ética jurídica, ya que forma parte de uno de los puntos del debate actual.

El debate surge en relación a que el uso de tecnologías que se consideran como cosas inanimadas, se tornan cada día más en su uso cotidiano y su ingreso en los temas de gobernanza se acerca con rapidez, por lo que, la necesidad de su regulación ética y jurídica, en el entendido de que el derecho no podrá realizarlo por su cuenta, ya que existe la necesidad de contar con conocimientos especializados en otras materias tal como lo señala Julio Ponce Solé "pero el Derecho no podrá hacerlo sólo, porque aqui la importancia de la transdisciplinariedad deviene crucial"16.

\footnotetext{
${ }^{14}$ Este fragmento forma parte del contenido de la Comisión Europea, en relación con la inteligencia artificial, citado con anterioridad.

${ }^{15}$ Ibidem.

${ }^{16}$ Fragmento tomado del articulo consultable en el portal web consultado con fecha 10 de Noviembre de 2019, laadministracionaldia.inap.es/noticia. asp?id=1509505\#nota2
} 
Lo complejo del presente debate gira entorno a la perspectiva ética y a la posibilidad material de concebir una inteligencia que pueda someter una consideración equivalente a un fallo judicial, esto en la temática derivada del derecho, de donde precisamente surge el problema que se pretende abordar y que paulatinamente ha ido encontrando su camino en la tecnología, al respecto Jhon Zeleznikow ${ }^{17}$ señala como las más importantes áreas de la IA, y el derecho a las siguientes:

a) Representación de la legislación con fines tanto de manutención de la misma, asi como de inferencia, por ejemplo, el trabajo realizado por Soft Law ${ }^{18}$.

b) Representación y razonamiento con conceptos jurídicos de textura abierta.

c) Desarrollo de ontologías jurídicas para mejorar el desempeño de los motores de búsqueda en la recuperación de información jurídica, asi como para tratar de uniformar el lenguaje jurídico, en la Red Mundial (World Wide Web o WWW).

d) Desarrollo de Sistemas de Asesoría Jurídica (Legal Advisory Systems), por ejemplo, el sistema Split-Up que asesora sobre la forma más probable en que se distribuirán los bienes, luego de la disolución del vínculo matrimonial.

e) Desarrollo de regulaciones y estatutos computarizados para mejorar la creación y recuperación de documentos legislativos.

f) Sistemas de Apoyo a la Decisión Jurídica (Legal Decision Support Systems), por ejemplo, el sistema Get Aid capaz de predecir el posible resultado de una controversia jurídica, y determinar si una solicitud de asistencia legal procede o no, o "Justiniano": prototipo de sistema experto en el ámbito de los derechos humanos que fue desarrollado en el IIJ de la UNAM ${ }^{19}$.

g) Desarrollo de Sistemas de Recuperación y Tratamiento de Documentación Jurídica (Legal Document Management and Retrieval Systems), por ejemplo, los sistemas australianos Datalex y Flex Law.

h) Desarrollo de sistemas para la argumentación y negociación jurídicas.

i) Tutores inteligentes, por ejemplo, el sistema CATO desarrollado por Kevin Ashley en Pittsburg, el cual presenta ejercicios a los estudiantes, los cuales incluyen los hechos del caso, un conjunto de precedentes en línea, e instrucciones acerca de cómo argumentar o contra argumentar.

\footnotetext{
17 Información obtenida del artículo publicado por Enrique Cáceres, Titulado Inteligencia Artificial, Derecho $Y$ Justica (El Proyecto lij-Conacyt), visible en el portal web https://revistas.juridicas.unam.mx/index.php/derecho-comparado/article/view/3889, consultado el 10 de Noviembre de 2019.

${ }^{18}$ Con la intención de conocer más respecto de esta temática, se recomienda la lectura del articulo EI Fenómeno Del Soft Law Y Las Nuevas Perspectivas Del Derecho Internacional, del autor Mauricio Ivan del Toro Huerta, visible en el siguiente portal web https://revistas.juridicas.unam.mx/index.php/derechointernacional/article/view/160/257

${ }^{19}$ Con la intención de conocer más respecto del presente sistema, se recomienda la consulta completa del sistema ofertado por la UNAM que resulta de sumo interés para la presente investigación, y que sin duda se vuelve un referente en la aplicación de derechos humanos y uso de sistemas tecnológicos en específico un sistema experto, se recomienda el seguimiento de dicho sistema mediante el portal web http://ru.juridicas.unam.mx/xmlui/handle/123456789/11411
} 
j) Desarrollo de sistemas que fomenten y promuevan la práctica del comercio electrónico (E-Commerce), por ejemplo, sistemas para la detección de páginas Web que pongan a la venta productos piratas.

Desde esta perspectiva encontramos dos tipos de postura en torno al uso de las tecnologías en el derecho, la primera se establece en torno a la ética y valores relacionados con el campo del derecho, y el segundo, a la eficiencia de los sistemas y el tipo de sistemas y tecnologías que deben usarse en la impartición de justicia y el uso de las tecnologías.

Tal y como se analizó brevemente desde el principio del presente artículo el uso de la tecnología ha formado parte en el origen del derecho "Desde el punto de vista de su uso, el derecho mismo -sus categorizaciones, sus procedimientos sus prácticas - constituye una tecnología cognitiva, un modo de aproximarse a la realidad social para controlarla, dirigirla y hacer efectivos en ella determinados planes y modelos económicos, sociales y políticos". ${ }^{20}$

En este sentido, la observancia del derecho no como un conjunto de normas derivado ya de un análisis de tecnología social, sino más bien su categorización desde una perspectiva cognitiva, cuyo objeto tiene que ver con el cumplimiento de determinados planes, no solo sociales, sino políticos y económicos, nos deja observar la necesidad de los Estados por permitir el uso de las más avanzadas herramientas matemáticas, sistemáticas, de digitalización y las que se encuentren a su alcance para lograr lo que debiera ser el tan anhelado acceso a la justicia.

Este reto se ha convertido en el estudio que emerge en la relación entre la sociedad y la tecnología, donde el tema transdisciplinario se vuelve fundamental para exprimir al máximo las posibilidades no solo sociales sino, sistemáticas y tecnológicas, que aporten la posibilidad de llegar con mayor rapidez y eficiencia a un objetivo y planteamiento en este caso en materia de impartición de justicia, para entrar en tema, corresponderá señalar las tecnologías que hoy rodean el derecho y que se están desarrollando, esto desde una perspectiva general.

Es claro que la concepción de los pensadores y juristas ilustrados del siglo XIX, como por ejemplo Josserand, "porque la industria se mejora y se transforma, es que los accidentes ocasionados o el hecho de las cosas inanimadas se tornan más numerosas, mucho más terribles y también mucho más obscuras. La injusticia del sistema tradicional de responsabilidad se ve claramente. En la inmensa mayoría de los casos, las víctimas se encuentran con la imposibilidad de reconstruir la génesis del accidente, de descubrir por qué y de demostrar la culpa del patrón de la gran industria, del conductor, etc." 21

${ }^{20}$ Casanovas. Pompeu, Derecho, Tecnología, Inteligencia Artificial Y Web Semántica. Un Mundo Para Todos Y Para Cada Uno" Texto extraído del Portal Web de la Universidad Nacional Autónoma de México, Instituto de Investigaciones Jurídicas. https://biblio.juridicas.unam.mx/bjv/detalle-libro/3875enciclopedia- de-filosofia-y-teoria-del-derecho-volumen-uno

${ }^{21}$ Ponce Sole, Julio, "Inteligencia artificial, Derecho administrativo y reserva de humanidad: algoritmos y procedimiento administrativo debido tecnológico" INAP, Instituto Nacional de Administración pública, del Ministerio de Política territorial y de función pública del Gobierno de España, el cual puede ser consultable en el siguiente portal web laadministracionaldia.inap.es/noticia.asp?id=1509505 
El derecho por su naturaleza tiene una "subestructura informacional" lo que según Richard E. Susskind denominó hace algún tiempo "I.- Oralidad (comunicación por medio del habla), II.- Escritura (simbolismo ideográfico o gramatical, III.- Imprenta (reproducción mecánica de la escritura y de la imagen), $I V$.- tecnologías de la Información automatización del modo de captura, compartir y comunicar información). ${ }^{22}$

Realmente la clasificación que se encuentra al alcance del conocimiento resulta bastante variada, esto se debe principalmente a que el enfoque que se toma respecto del uso de los sistemas y las tecnologías, por lo que los puntos ideológicos y argumentativos que las sustentan serán diversos según el tipo de sistema utilizado a continuación se señalan los sistemas expuestos por dos autores Pampeu Casanovas y Juan Antonio Enríquez, a saber:

Los Sistemas multiagentes (Multi-Agent Systems, MAS); Las Instrucciones electrónicas o instituciones virtuales; III.- Los sistemas computacionales dialógicos. - IV.- Derecho relacional (Relational law). V.- Que afectan los sistemas de mediación, negociación, gestión y resolución de conflictos, Los antes mencionados son los establecidos por el autor Pampeu Casanovas. ${ }^{23}$

Así como los expuestos por Juan Antonio Enríquez Solís ${ }^{24}$, I.- Sistemas abiertos, II.- Sistemas cerrados o absolutamente aislados III.- Sistemas apoteóticos IV.Sistemas emergentes.

Como se ha hecho mención este ensayo versará sobre la perspectiva de las herramientas tecnológicas que se están haciendo valer para la impartición de justicia y la toma de decisiones, pero no podemos desvincularlo en su totalidad del agente ético, ya que su intrínseca relación con la humanidad, nos conduce a vislumbrarlo siempre desde la perspectiva no solo tecnológica sino del espíritu humano y ceñirlo desde lo intangiblemente del ser, y dejar claro que el debate de las máquinas nunca deberá encontrarse fuera del debate de lo humano.

\section{LA ÉTICA Y LÓGICA EN EL DEBATE DEL USO DE LAS TECNOLOGÍAS EN EL DERECHO}

En primera instancia se define qué es la ética para uso práctico en este apartado. El término ética se deriva de la palabra griega ethos. Parece ser que el primero que usó esta palabra fue el poeta Hornero, quien entendía esta palabra como: "Lugar

${ }^{22}$ Referencia tomada del texto Derecho, Tecnología, Inteligencia Artificial Y Web Semántica. Un Mundo Para Todos Y Para Cada Uno", op.cit. p. 826.

${ }^{23}$ Para la comprensión del tema se recomienda la lectura del texto "Derecho, Tecnología, Inteligencia Artificial y Web Semántica, un mundo para todos y para cada uno, del autor Pompeu Casanovas, visible en el portal web https://archivos.juridicas.unam.mx/www/bjv/libros/8/3875/26.pdf. Consultado con fecha $12 / 05 / 2021$.

${ }^{24}$ Autor de la Tesis "Derecho Y Lógica Fuzzy, Una Aproximación Desde La Cibernética Y La Inteligencia Artificial",de la Universidad Nacional Autónoma de México, publicada en 2019, y que puede ser consultable en el portal web oficial de la UNAM http://dgb.unam.mx/index.php/catalogos/librunam. 
habitado por hombres y animales". En este sentido primigenio, un pensador contemporáneo, Martin Heidegger, se refiere a este vocablo como lugar o morada, y por ello dice que la morada o ethos del hombre es el ser. ${ }^{25}$

Existe una gran controversia respecto a las nuevas tecnologías en cuestiones de ética, en el caso de la Digitalización y los Algoritmos Sistemáticos (AID), citando a Adolfo Plasencia: "Si se trata de humanos, la ética debe existir ya que hablamos de un entorno fundamentalmente intelectual... Eso no significa que los modos de ejercerla y de aplicarla sean los mismos, aunque la ética humana sea de la misma naturaleza. ${ }^{26,}$

Podemos constatar que al momento de hablar sobre ética referente a la digitalización nos posiciona en una gran disyuntiva, debido a que al tratarse del ser humano queda intrínseco que la ética debe estar presente, la cuestión es ¿cuáles son los modos que se emplearán para hacerla ejercer?

Una materia que ayudará en desarrollo en este rubro son las matemáticas, específicamente la lógica matemática que como se menciona a continuación:

“...El hombre requirió desde el principio de la civilización de procesos que le permitieran comprender y obtener conclusiones precisas para transformarse a sí mismo y a su entorno natural. Este proceso, que marca la diferencia con respecto de los demás seres vivos, es la capacidad de razonamiento. En el ánimo de perfeccionar esta capacidad la historia de las diversas culturas nos da cuenta de cómo se inició el estudio de los medios para desarrollarla. Hemos señalado en forma somera la evolución de la matemática, y la lógica se encuentra intimamente ligada a ésta.

Puede definirse a la lógica como el estudio de los métodos y principios usados para distinguir el razonamiento correcto del incorrecto. En el ámbito jurídico, que a nosotros interesa, la lógica es un instrumento importante para la interpretación y aplicación del derecho. Por ello, analizaremos en forma somera los razonamientos lógicos inductivo, deductivo y analógico, destacando que strictu sensu no se trata de métodos sino de formas de pensamiento o razonamiento... "27

De lo anterior se desprende la evolución histórica del pensamiento humano desde los tiempos más remotos hasta nuestros días, y hemos visto a lo largo de los siglos como el hombre no solo se hizo sedentario, sino que, como homo faber transformó su medio ambiente circundante y creo utensilios, herramientas y grandes sistemas hidráulicos y, más tarde, en esa evolución histórica, ya como homo sapiens, creo la filosofía y otras disciplinas del pensamiento hasta llegar a nuestros días, a esta era maravillosa de las tecnologías.

\footnotetext{
${ }^{25}$ Escobar Valenzuela y Luis Amador Valdez Vázquez, ÉTICA. 4th ed. México, D.F (año 2000. p.43.

26 Plasencia, A. (2015). Ética, Digitalización y Social Media. [online] El Mundo. portal web https://www.elmundo.es/economia/2015/03/02/54f4c6b8268e3e9e4b8b456b.html consultada el día 10 de Noviembre 2019.33

${ }_{27}$ Cienfuegos Salgado, D. (2004). Matemáticas Aplicadas al Derecho. 2nd ed. México, DF: Editorial Porrúa. Sa De Cv, pp.7-10
} 
A continuación, abordaremos las definiciones que mantiene la lógica para delimitar la inclusión de esta para abarcar el tema de la ética con lo siguiente:

Razonamiento Inductivo: Es la forma de razonamiento en la que, a partir de un número de observaciones particulares, se concluyen leyes generales. Como señala Ponce de León, el método inductivo, considera una serie de fenómenos o conocimientos particulares para llegar a conclusiones generales. Esta forma de razonamiento es más usual en el trabajo de laboratorio donde se observan y experimentan diversos hechos propios de la física, la química, la biología, la psicología, etcétera. En el ámbito jurídico encontramos que el método inductivo se puede instrumentar de muy diversas formas, pero principalmente mediante las técnicas de análisis y presentación de casos, de procesos jurídicos, de resoluciones jurisdiccionales y jurisprudenciales, etcétera.

Razonamiento deductivo: Es la forma de razonamiento en que concluimos ciertos principios o conocimientos particulares a partir de principios o conocimientos generales. Esta forma de pensamiento es ideal para realizar investigaciones en las ciencias formales. En materia jurídica, señala Ponce de León, el método deductivo se realiza principalmente mediante las técnicas de aplicación de las normas jurídicas generales a casos concretos.

El razonamiento deductivo tiene un marcado interés para el derecho, pues durante mucho tiempo esta forma se ha aplicado en la resolución de casos. Ello mediante el uso de silogismos en las resoluciones judiciales. Aunque no puede predicarse que esa sea la forma adecuada de resolución de problemas, lo cierto es que el uso de silogismos ilustra a la perfección el razonamiento de los operadores jurídicos tradicionales. Un silogismo razonamiento deductivo es en el que se infiere una conclusión de dos premisas.

Razonamiento analógico: Es la forma de pensamiento en la cual la conclusión tiene el mismo grado de particularidad o generalidad que sus premisas. Esta forma de razonar es considerada por algunos autores como una derivación del pensamiento inductivo, por la discutible certeza de sus conclusiones. Como menciona Ponce de León, este método consiste en la comparación de fenómenos por sus semejanzas y diferencias, yendo de lo conocido a lo desconocido. En el contexto jurídico puede aplicarse en la modificación legislativa y en la elaboración de normas jurídicas, para lo cual conviene siempre considerar la experiencia normativa en el tiempo y en el espacio, situación que origina la comparación histórica y la comparación sociológica ${ }^{28}$.

$\mathrm{Al}$ analizar los razonamientos inmediatos anteriores, podemos concluir que a través de estos el hombre se ha acercado al conocimiento del derecho desde el punto de vista metodológico utilizando el razonamiento para establecer verdades lógicas a partir de la observación y el análisis del fenómeno jurídico.

Como se ha venido desarrollando en el presente ensayo, para aprender respecto del tema que se presenta, debe primero desaprenderse de los temas que hoy invaden

\footnotetext{
${ }^{28}$ Ibidem.
} 
el derecho como una ciencia o un arte sin adentrarnos al debate, para verlos desde una nueva perspectiva, cuya base es la lógica y que puede ser traducida en un nuevo lenguaje, esto desde la óptica que se tiene de este instrumento como una tecnología cognitiva, que hoy puede ser estudiada y combinada con diversos sistemas, instrumentos y ramas del conocimiento humano.

Seguro es para quien desconozca las ciencias exactas, el comprender que determinadas hipótesis llevadas en la convivencia social, pueden ser ya determinadas por sistemas algorítmicos y matemáticos, el sentido que se debe comprender, es que el derecho como bien sabemos es un sistema regulador, por lo que este sistema no es más que delimitaciones y posibilidades sugeridas por un estado con determinados fines, y las aporías que ante él se presentan con regularidad se sustentan en hipótesis normativas específicas, desde un rango constitucional hasta un rango reglamentario, desde una teoría hasta una jurisprudencia, e incluso, datos históricos y todas esas alternativas pueden ser sin duda medibles gracias a la tecnología.

Para comprender este tema se debe salir del horizonte de las letras para adentrarse al mundo numérico, cuya determinación tiene una cabida muy relevante en nuestra sociedad no solo actual sino histórica; a saber, las ciencias matemáticas han surgido con la humanidad desde muy temprano, inclusive, marcando, a las civilizaciones cuyos avances matemáticos eran sobresalientes como los pueblos más importantes, y es el caso que las tecnologías cognitivas permiten determinar una serie de posibilidades previamente registradas, para obtener el resultado más óptimo en la solución de un problema.

Ahora bien y derivado precisamente de que el presente ensayo busca desincorporar los conceptos tradicionales del estudio del derecho, meramente con una perspectiva argumentativa que si bien encuentra un sustento bastante interesante y para algunos rebuscado, lo cierto es que desde ahora se plantean desde determinadas situaciones a partir de un plano netamente cuantitativa, esto derivado de que el tema de nuestro ensayo guarda estricta relación con la sistematización y el uso de la tecnología, y dado que son precisamente asignaturas relacionados con los sistemas informáticos y estos derivan de ordenes algorítmicas, ha sido importante diseñar el trabajo en esta línea del conocimiento.

Es claro que esta forma de ver el derecho no resulta innovadora, inclusive las teorías de la lógica argumentativa que hoy día se plasman por los ius filósofos contemporáneos como Manuel Atienza, se ciñen en explicar su razonamiento a través de fórmulas, verbo y gracia el texto "Las Razones del Derecho", "Teorías de la argumentación jurídica" ${ }^{\prime 29}$, en donde el autor, desde el año 2005, formula una propuesta del uso de fórmulas para la comprensión de las premisas, que se traducen y se observan en su texto de la siguiente forma:

\footnotetext{
${ }^{29}$ Atienza, Manuel. Las Razones del Derecho, Teorías de la argumentación jurídica, Universidad Nacional Autónoma de México, 2005, texto consultado con fecha 10 de Noviembre de 2019 y verificable completamente en el portal web perso.unifr.ch/derechopenal/assets/files/obrasjuridicas/oj_20151108_01.pdf
} 
a) Todos los poetas son imbéciles. El ministro es un poeta. Por tanto, el ministro es un imbécil.

En la logica proposicional, la inferencia podría representarse aproximadamente (cfr. infra, cap. V, apartado 3.1.2), asi:

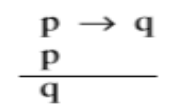

Una vez explicado que el desarrollo de este ensayo si bien guarda relación con el derecho, hay que abrir paso para abordar el tema desde una óptica matemática, y la ética no escapará de dicha óptica, en el aspecto de que todo se enlaza precisamente con la cognición humana y la lógica ancestral que deriva estrictamente del estudio filosófico que se tiene respecto de la ética y la lógica, la segunda es sin duda el cimiento mismo de las matemáticas, e incluso de los argumentos que se esgriman en nuestra profesión, para poner un ejemplo de la lógica cabe señalar:

La lógica es la ciencia que estudia el razonamiento, donde "razonar" consiste en obtener afirmaciones (llamadas conclusiones) a partir de otras afirmaciones (llamadas premisas) con los criterios adecuados para que podamos tener la garantía de que si las premisas son verdaderas, entonces las conclusiones obtenidas también tienen que serlo necesariamente. Por ejemplo,

Todos los españoles son europeos,

Cervantes era español,

luego Cervantes era europeo ${ }^{30}$.

Claro está que el presente ensayo de ninguna manera innova en el uso algorítmico y matemático. En el derecho existen diversos autores que se han encargado de tocar el tema, podemos mencionar a Rosario Delgado quien en Barcelona ha escrito respecto de la probabilidad y el derecho, esto desde una perspectiva meramente matemática, explicando en su texto Derecho y probabilidad: Falacias, fórmula de Bayes y redes Bayesianas ${ }^{, 31}$, en donde las fórmulas pasan a ser parte de la probabilidad de comisión de un ilícito.

En este sentido, como se ha podido apreciar, el lenguaje usado en el presente ensayo estima su cercanía con fórmulas matemáticas y algoritmos, dichas fórmulas son precisamente el lenguaje de la programación y es lo que demuestra la cercanía entre el pensamiento y las matemáticas ahora en las ciencias sociales o tradicionalmente llamadas ciencias del espiritu ${ }^{32}$.

\footnotetext{
${ }^{30}$ Ivorra Castillo, Carlos. La Lógica Matemática. Pag. IX.El texto completo puede ser consultado en el portal web https://www.uv.es/ivorra/Libros/Logica2.pdf, Consultado el 10 de Noviembre de 2019.

${ }^{31}$ Se recomienda la lectura del texto el cual puede ser encontrado en línea y consultado el 11 de Noviembre de 2019 en el portal web http://mat.uab.cat/matmat/PDFv2013/v2013n06.pdf

${ }^{32}$ Dilthey, Wilhelm, Introducción a las Ciencias del Espiritu, Fondo de Cultura Económica, el cual puede ser consultado en el portal web www.posgrado.unam.mx/filosofia/pdfs/Textos_2019-1/20191_Dilthey_IntroduccionCienciasEspiritu.pdf, consultado el 12 de Noviembre de 2019.
} 
En este sentido la ética, también puede analizarse desde una perspectiva lógica, bajo el método de inducción; lo cual se definirá a continuación:

Para el método inductivo de la ética se utiliza:

El esquema lógico que corresponde a la inducción es el siguiente:

$A, B, C \ldots$ son $S$

$A, B, C \ldots$ son $P$

Todo $S$ es $P^{33}$

Para reforzar lo postulado anteriormente de manera cuantitativa dando conexión con la digitalización y los Algoritmos Sistemáticos (AID) referente a la ética es con el método de inducción:

Haciendo énfasis en dos características fundamentales del conjunto de los números naturales: en primer lugar, los naturales forman un conjunto infinito, ordenado y que cuenta con un primer elemento (el número 1) y, en segundo lugar, que el resto de los elementos del conjunto (los demás naturales) se generan a partir del número $\mathbf{1}$ mediante repetidas aplicaciones de la función sucesor: $\mathbf{s}(\mathbf{n})=\boldsymbol{n}+\mathbf{1}$.

Las propiedades anteriores son fundamentales, pues de ellas se derivan las bases lógicas que dan sustento a la validez de las demostraciones por Inducción. En su forma más básica, el método de Inducción Matemática consta de 2 etapas. Si deseamos probar que una determinada propiedad $\boldsymbol{P}$ se cumple para todo número natural, entonces procedemos aplicando el siguiente esquema:

- Paso 1 (Base de la Inducción).- Se demuestra que el primer natural (el número 1) cumple la propiedad: $\mathbf{P ( 1 )}$.

- Paso 2 (Paso Inductivo).- Partiendo de la suposición (Hipótesis de Inducción) de que un número natural cualquiera $\mathbf{k}$ cumple con la propiedad: $P(k)$, procedemos a demostrar que, en consecuencia, el número $\boldsymbol{k}+\mathbf{1}$ también debe cumplir con dicha propiedad: $\mathbf{P}(\boldsymbol{k}+\mathbf{1})$. Es decir, probamos la validez de la implicación $\mathbf{P}(\mathbf{k}) \Rightarrow \mathbf{P}(\boldsymbol{k}+\mathbf{1})$.

En caso de poder realizar exitosamente lo anterior, entonces concluimos que la propiedad $\mathbf{P}$ se cumple para todos los números naturales. A continuación, veremos cómo funciona el método...

$$
1+2+\cdots+n=\frac{n(n+1)}{2} .
$$

Paso 1 (Base de la Inducción).- Procedemos a mostrar la validez de la fórmula para el caso $\boldsymbol{n}=\mathbf{1}$.

$$
1=\frac{1(1+1)}{2}=\frac{2}{2}=1
$$

- Paso 2 (Paso Inductivo).- Suponemos que la fórmula es válida para un número natural cualquiera $\boldsymbol{n}=\boldsymbol{k}$.

Hipótesis de Inducción:

$$
1+2+\cdots+k=\frac{k(k+1)}{2}
$$

\footnotetext{
${ }^{33}$ Escobar Valenzuela op cit.
} 
European Journal of Social Law

$$
\begin{aligned}
& y \text { a partir de esto establecemos la validez de la fórmula para } \boldsymbol{n}=\boldsymbol{k}+1 .^{34} \\
& 1+2+\cdots+(k+1)=(1+2+\cdots+k)+(k+1) \\
&=\frac{k(k+1)}{2}+k+1 \\
&=\frac{k(k+1)+2(k+1)}{2} \\
&=\frac{(k+1)(k+2)}{2} \\
&=\frac{(k+1)[(k+1)+1]}{2}
\end{aligned}
$$

Desglosando lo anterior, se demuestra mediante cálculos matemáticos la relación que tiene un postulado con el anterior. Manteniendo la perspectiva de la idea del método inductivo de la ética se puede traducir de forma matemática por el método de inducción cómo herramienta para los algoritmos sistemáticos, siguiendo principalmente la lógica del planteamiento anterior.

Sin duda, a partir de estas ideas, podemos incluir en la ecuación a la Inteligencia Artificial, mediante los algoritmos sistemáticos incluyendo lo postulado en el párrafo anterior, la ética puede ser aprehendida por la inteligencia artificial si en sus algoritmos se implanta dicha lógica, un supuesto, lo que precisa del dilema actual entorno a la forma con mayor probabilidad de éxito o mejores resultados de eficiencia.

A pesar de lo anterior, puede surgir aún la interrogante ¿cómo se podría prevenir que la Inteligencia Artificial no caiga en el estereotipo distópico? Un matemáticofilósofo británico llamado Alan Turing propuso en 1950 una prueba para confirmar si una computadora pudiera ser capaz de razonar (origen de la idea de la Inteligencia Artificial), llamado The Turing Test.

El cual se compone de la siguiente manera: un computador y dos personas reales. Tanto $A$ (computador) como $B$ (persona real), responden a las preguntas formuladas por $C$ (juez), quien debe identificar si está hablando con dos personas o si una es un computador. Mientras que la persona responde a las preguntas de manera veraz, el computador debe responder cómo si fuese un humano, de modo que $C$ crea que A también es una persona. Para evitar que se descubra de manera obvia quién es quién, las preguntas y respuestas son escritas e impresas a través de tele-impresoras. Si A es lo suficientemente convincente, C concluirá que hay dos personas en vez de una, lo cual revela el peculiar papel que A desempeña, a saber, debe causar que C se equivoque en la identificación. ${ }^{35}$

De acuerdo con lo mencionado, podemos observar que al momento de realizar la prueba las preguntas que se plantean sean referentes a la ética para analizar si el algoritmo funciona y la Inteligencia Artificial es "ética".

\footnotetext{
${ }^{34}$ Ruiz Benjumeda, F). "Demostrando por Inducción”. Revista Tzaloa, [online] (3), pp.2 y 3. Available at: http://www.ommenlinea.org/wp-content/uploads/2014/01/09-3.pdf información verificada con fecha10 de Noviembre de 2019.

${ }^{35}$ Hayes, P. and Ford, K. (n.d.). Turing Test Considered Harmful. Consultado con fecha 10 de Noviembre de 2019, en el portal web at:https://www.researchgate.net/profile/Kenneth_Ford/publication/ 220813820_Turing_Test_Considered_Harmful/links/09e4150d1dc67df32c000000.pdf
} 
Esta postura que deviene de un análisis matemático también puede contraponerse con la complejidad de la determinación de posibilidades que pueden darse en la ética que deviene del ser humano, la cual ha visto transformación desde sus remotos orígenes. En este debate respecto de que, si la lógica matemática deba o no tener relación con las decisiones en torno a la estabilidad de una sociedad o incluso determinen política pública, hay que señalar que actualmente existen sistemas estadísticos que determinan instituciones gubernamentales tales como el INEGI ${ }^{36}$, cuyas encuestas se encuentran sometidas a sistemas tecnológicos de digitalización.

Desde esta perspectiva, se está presentando como síntoma del uso de esta revolución o transformación de la forma de presentar la verdad científica respecto de un tema inclusive que guarde relación con el futuro de la humanidad, las acciones y medidas a tomar.

Esto nos presenta el debate actual del uso de la tecnología y la responsabilidad que recae en esta generación de la humanidad, en donde derivado de lo trascendentes que pueden ser los cambios en una sociedad y la rapidez y fluidez con la que se transmiten los fenómenos en espacios geográficos, lo que conlleva a obtener avances en menor tiempo, en casi todo el planeta, por lo que este fenómeno hoy estudiado denominado globalización, resulta ser piedra angular del debate.

Entrando en un tema filosófico, la Inteligencia Artificial al trabajar en conjunto con los nuevos sistemas y plataformas de operación, podemos observar que se está alimentando con nuevas tecnologías al concepto de un "Ser Autónomo" que sea capaz de analizar de forma ajena al ser humano, le dé esa perspectiva que necesita la humanidad para poder (R)evolucionar.

Y no es para menos, las nuevas tecnologías aportarán al ser humano en su travesía por la experiencia que llamamos vida; optimizando, reformando, contrastando, todo desde una perspectiva lógica, a la par del conocimiento humano. Un guía que apoye a la "ética", anteponiendo los valores esenciales para una convivencia civilizada y le permita a la humanidad expandir su panorama a nuevas cuestiones, ya que sus necesidades básicas serán cubiertas.

\section{CONCLUSIONES}

Sin duda las actividades humanas se han diversificado y los espacios digitales ocupan las enormes plataformas creadas para su funcionamiento, sobre todo en los últimos meses, debido a la pandemia, nos hemos vuelto más dependientes de las Tics en el mundo; por ello es importante entender la revolución del derecho informático y la Informática jurídica en este nuevo mundo globalizado.

\footnotetext{
${ }^{36}$ Instituto Nacional de Estadística, Geografía e Información, Organismo Autónomo del Gobierno de México. www.inegi.org.mx
} 


\section{FUENTES DE INVESTIGACIÓN:}

- Casanovas. Pompeu, Derecho, Tecnología, Inteligencia Artificial Y Web Semántica. Un Mundo Para Todos Y Para Cada Uno" Texto extraído del Portal Web de la Universidad Nacional Autónoma de México, Instituto de Investigaciones Jurídicas. https://biblio.juridicas.unam.mx/bjv/detalle-libro/3875-enciclopedia-de-filosofia-y-teoria-delderecho-volumen-uno.

- Guibourg, Ricardo. Informática Jurídica, UNAM, consultable en el portal web consultado con fecha: 4 Diciembre 2019 https://archivos.juridicas.unam.mx/www/bjv/libros/8/3875/25.pdf

- Rodríguez, Felipe. "Lecciones de derecho y ética para ingenieros, estudiantes de ingeniería $y$ profesiones afines" UNC, consultable en su totalidad en el portal web www.feliperodriguez.com.ar/wp-content/uploads/2013/11/LIBRO-7-DERECHOINFORMATICO.pdf , consultado con fecha 4 de Diciembre de 2019.

- Gustavino, Elías "Responsabilidad civil y otros problemas jurídicos en computación”, Ed. La Rocca, 1987.

- Fundamentos de Informática $I$, Conceptos generales, consultable en el portal web. https://www.infor.uva.es/ cevp/FI_I/fichs_pdf_teo/FI_I_Tema1.pdf, consultado con fecha 10 de Diciembre de 2019.

- Definir la orientación y las prioridades políticas generales de la Unión Europa, para mejor comprensión de dicho consejo se recomienda la búsqueda y seguimiento de dicho consejo mediante su portal web oficial consultado con fecha 10 de noviembre de 2019. https://europa.eu/european-union/about-eu/institutions-bodies/european-council_es

- Enrique Caceres, Titulado Inteligencia Artificial, Derecho Y Justica (El Proyecto lij-Conacyt), visible en el portal web https://revistas.juridicas.unam.mx/index.php/derechocomparado/article/view/3889, consultado el 10 de Noviembre de 2019.

- El Fenómeno Del Soft Law Y Las Nuevas Perspectivas Del Derecho Internacional, del autor Mauricio Ivan del Toro Huerta, visible en el siguiente portal web https://revistas.juridicas.unam.mx/index.php/derecho-internacional/article/view/160/257

- Ponce Sole, Julio, "Inteligencia artificial, Derecho administrativo y reserva de humanidad: algoritmos y procedimiento administrativo debido tecnológico" INAP, Instituto Nacional de Administración pública, del Ministerio de Política territorial y de función pública del Gobierno de España, el cual puede ser consultable en el siguiente portal web laadministracionaldia.inap.es/noticia.asp?id=1509505

- Autor de la Tesis "Derecho Y Lógica Fuzzy, Una Aproximación Desde La Cibernética Y La Inteligencia Artificial", de la Universidad Nacional Autónoma de México, publicada en 2019, y que puede ser consultable en el portal web oficial de la UNAM http://dgb.unam.mx/index.php/catalogos/librunam.

- Escobar Valenzuela y Luis Amador Valdez Vázquez, ÉTICA. 4th ed. México, D.F (año 2000. p.43.

- Plasencia, A. (2015). Ética, Digitalización y Social Media. [online] El Mundo. portal web https://www.elmundo.es/economia/2015/03/02/54f4c6b8268e3e9e4b8b456b.html consultada el día 10 de Noviembre 2019.

- Cienfuegos Salgado, D. (2004). Matemáticas Aplicadas al Derecho. 2nd ed. México, DF: Editorial Porrúa. Sa De Cv, pp.7-10

- Atienza, Manuel. Las Razones del Derecho, Teorías de la argumentación jurídica, Universidad Nacional Autónoma de México, 2005, texto consultado con fecha 10 de Noviembre de 2019 y verificable completamente en el portal web perso.unifr.ch/derechopenal/assets/files/obrasjuridicas/oj_20151108_01.pdf 
- Ivorra Castillo, Carlos. La Lógica Matemática. Pag. IX.El texto completo puede ser consultado en el portal web https://www.uv.es/ivorra/Libros/Logica2.pdf, Consultado el 10 de Noviembre de 2019.-

- Dilthey, Wilhelm, Introducción a las Ciencias del Espiritu, Fondo de Cultura Económica, el cual puede ser consultado en el portal web www.posgrado.unam.mx/filosofia/pdfs/Textos_20191/2019-1_Dilthey_IntroduccionCienciasEspiritu.pdf, consultado el 12 de Noviembre de 2019.

- Ruiz Benjumeda, F). "Demostrando por Inducción". Revista Tzaloa, [online] (3), pp.2 у 3. Available at: http://www.ommenlinea.org/wp-content/uploads/2014/01/09-3.pdf información verificada con fecha10 de Noviembre de 2019.

- Hayes, P. and Ford, K. (n.d.). Turing Test Considered Harmful. Consultado con fecha 10 de Noviembre de 2019, en el portal web at:https://www.researchgate.net/profile/Kenneth_Ford/ publication/220813820_Turing_Te 